\title{
Criminalidad, inseguridad pública y comportamiento de los electores: un análisis del proceso electoral estatal 2010 en Ciudad Juárez, Chihuahua \\ Andrés Valdez Zepeda*, Abraham Paniagua Vázquez ${ }^{* *}$
}

\section{Resumen}

En este escrito se hace un análisis del efecto de la crisis de inseguridad pública derivada de la llamada "guerra contra el narcotráfico" en la conducta del votante en un momento electoral, a la luz de las diferentes teorías sobre el voto. Se describe en lo particular el efecto de la actual crisis de inseguridad pública en los resultados de las elecciones locales del 2010 en Ciudad Juárez, Chihuahua, México. Se concluye que una crisis de inseguridad y violencia orienta el comportamiento electoral. El caso de Ciudad Juárez muestra que a pesar de los indicadores de inseguridad pública y la insurgencia criminal, el Partido Revolucionario Institucional, como partido gobernante a nivel municipal y estatal, logró ganar la elección.

Palabras clave: inseguridad pública, conducta del elector, violencia, elecciones 2010, Ciudad Juárez, teorías del voto.

\begin{abstract}
This text analyses the effect of the insecurity crisis as a result of the "war against narcotrafic" in the behaviour of citizens in election times. We particulary describe the effect of the current public safety crisis in the results of the local elections in Ciudad Juarez in 2010. We conclude that violence and insecurity crisis oriented voting behavior. Ciudad Juarez case shows that, despite public insecurity and violence indicators, the Partido Revolucionario Institucional, as the local and national ruling party, won the election.

Keywords: crisis of insecurity, conduct of the voter, violence, elections 2010, Juarez City, theories of vote.
\end{abstract}

* Doctor en Estudios Latinoamericanos con especialidad en Ciencia Política, actualmente trabaja como profesor-investigador en la Universidad de Guadalajara y en el Instituto Tecnológico y de Estudios Superiores de Occidente. Miembro del Sistema Nacional de Investigadores desde 1998. Correo electrónico: azepeda@cucea. udg.mx

** Doctor en Ciencias Sociales con especialidad en Gobierno y Asuntos Públicos, se desempeña como profesor investigador de la Universidad Autónoma de Chihuahua, Facultad de Ciencias Políticas y Sociales. Miembro del Sistema Nacional de Investigadores. Correo electrónico: apaniagua@uach.mx 


\section{Introducción}

América Latina está enfrentando una severa crisis de seguridad pública, manifiesta en el incremento sustancial del índice de inseguridad, violencia, comisión de delitos y ejecuciones, así como por la proliferación de peligrosos grupos de criminales cuyo modus operandi se caracteriza por ser transnacional. Al respecto, se ha vuelto un lugar común afirmar que en América Latina las células pertenecientes al crimen organizado han diversificado sus operaciones llegando a abarcar el tráfico de armas, el lavado de dinero, el secuestro, la extorsión y por supuesto el comercio ilícito de drogas, que generan a la vez ilegalidad, corrupción e impunidad que sirven de incentivo para generar los alarmantes índices de actividades delictivas.

Para el caso de México en general, la inseguridad ha alcanzado niveles sin precedentes. En los últimos años el país ha visto amenazada seriamente la paz social y la convivencia ciudadana se ha trastocado ante el aumento significativo de los asesinatos, secuestros, robos y extorsiones. De acuerdo con la Organización de las Naciones Unidas (onU, 2009), México ocupa el quinto lugar mundial en delincuencia y el treceavo lugar en delitos del fuero común. Las causas de dicho incremento son diversas, y van desde la pobreza y marginación en la que viven millones de mexicanos, la desintegración familiar, la falta de oportunidades de estudio y trabajo para millones de jóvenes, ${ }^{1}$ la impunidad, la corrupción de los cuerpos policiacos y la falta de estrategias de seguridad exitosas, hasta la "alta rentabilidad" que generan las actividades criminales, entre otras.

Esta crisis ha puesto en entredicho la función constitucional del Estado de otorgar seguridad pública a sus habitantes, trastocando también la legitimidad de los gobernantes en turno ante el ascenso de la violencia, la inseguridad y el crimen. El debilitamiento de facto del Estado y su men-

\footnotetext{
${ }^{1}$ La escalada de los indicadores de violencia y criminalidad en todo el país han puesto como foco de atención a los jóvenes que no tienen oportunidad de estudiar y trabajar, los llamados Ninis. La Encuesta Nacional de la Juventud 2005 concluye que 22\% de los jóvenes de 12 a 29 años no estudia ni trabaja (Imjuve, 2005). Ese porcentaje equivale a 7.5 millones de jóvenes. Por su parte, según la Encuesta Nacional de Ocupación y Empleo (ENOE) del segundo trimestre de 2009, sólo $1.4 \%$ de la población joven no estudia ni trabaja, lo que equivale a 285 mil jóvenes (INEGI, 2009).
} 
guante capacidad de gobierno se entiende para explicar el desorbitado ascenso en la actividad criminal (Serrano y Toro, 2005).

Entre la sociedad mexicana, la opinión generalizada es que los esfuerzos públicos por erradicar la actividad criminal y disminuir los efectos colaterales de la "guerra contra el narcotráfico" están condenados al fracaso debido a la mala preparación de los cuerpos policiacos, la marcada corrupción entre los actores públicos encargados de aplicar la ley, las sospechas de que la esfera política se ha beneficiado del narcotráfico y sobre todo por la extraordinaria penetración que el crimen organizado ha desarrollado en el tejido social. De esta manera ha llegado a su fin la percepción de que en México el narcotráfico, si bien generaba un mundo violento, éste se desarrollaba de manera autónoma, con sus propias leyes (Serrano y Toro, 2005).

El presente trabajo busca indagar sobre los efectos o impactos que la crisis de inseguridad pública que sufre esta ciudad pudo generar en el comportamiento y conducta del elector. Es decir, el objetivo de la investigación se orientó a tratar de conocer la relación que existe entre la crisis de violencia extrema y la orientación del voto de los electores en Ciudad Juárez, Chihuahua, México.

La pregunta general sobre la cual esta investigación ahonda es: ¿Existe relación o no entre la percepción social sobre la crisis de inseguridad pública en Ciudad Juárez y el comportamiento de los electores? Si existe, ¿qué tipo de relación es? A partir de aquí se generan otras dos interrogantes: ¿Cuál es el costo electoral para el partido gobernante y para la oposición política?, y ¿qué ventajas o beneficios políticos les generaron la crisis de inseguridad pública a los principales partidos y candidatos que contendieron en las elecciones?

La idea principal que guía este trabajo es que el contexto social enmarcado por la inseguridad pública configura la decisión en materia electoral. Es decir, la afectación o percepción sobre la existencia de la crisis de inseguridad pública genera niveles de malestar e incertidumbre entre los electores que a su vez se reflejan en la intención de hacer o no hacer efectivo el derecho a votar, y en su caso, orienta la decisión de por quién hacerlo. La idea principal dio pie al surgimiento de dos apuestas adicionales:

a) La existencia de la crisis de inseguridad pública genera ciertos niveles de malestar y miedo entre los electores, lo cual, en un momento electoral, 
ayuda a los partidos de oposición a aumentar el porcentaje de votos obtenidos en una elección y reduce el porcentaje de votos que obtiene el partido gobernante.

b) La existencia de la crisis de inseguridad pública no genera votos ni a favor de la oposición ni en contra del partido en el gobierno ya que la decisión de los votantes, en un momento electoral, responde a otro tipo de motivaciones de carácter político y económico.

Para esta investigación se procedió a realizar, en un primer momento, una revisión bibliográfica sobre las teorías que explican el comportamiento del elector, enfatizando en las que explican el comportamiento del votante a partir de consideraciones de carácter racional y emocional.

En un segundo momento se aplicó una encuesta preelectoral representativa entre 394 personas en condiciones de sufragar en Ciudad Juárez, Chihuahua, entre los días 10 y 15 de abril del 2010 (79 días antes de la elección constitucional) para saber su opinión sobre la crisis de violencia y su vinculación con su comportamiento electoral. De acuerdo con los resultados preliminares del Censo de Población y Vivienda (INEgI, 2010), en el 2010 la localidad fronteriza de Juárez contaba con 1,328,017 habitantes, de éstos, 1,055,761 se encontraban inscritos en la lista nominal del Instituto Estatal Electoral de Chihuahua (IEE, 2010). Se omitió tomar la opinión de aquellos ciudadanos que dijeron militar en un partido político ya que se considera que su militancia predefine de cierta forma su comportamiento electoral. Se consideró un error estadístico de $+-5 \%$ y una confiabilidad del 95\% (Scheaffer, 1987; Lohr, 2000). La selección de los entrevistados se determinó mediante una muestra aleatoria por conglomerados llevada a cabo en los siete distritos electorales locales (II, III, IV, V, VI, VII y VIII) situados en el municipio de Juárez.

Para tal efecto, el cuestionario se estructuró en cuatro partes: la primera asienta las características sociodemográficas; la segunda ahonda sobre las intenciones de votar o no votar y sus razones; la tercera explora la opinión de los encuestados sobre la relación inseguridad pública y comportamiento electoral; mientras que la cuarta incluye las preguntas relativas a la confianza en el voto, los partidos políticos, los candidatos y los gobiernos, siempre presente la inseguridad pública. De esta manera los resultados 
captaron una muestra representativa de las características generales de la población del municipio de Juárez.

El análisis estadístico contempló dos elementos: la descripción porcentual y un análisis de prueba de independencia de variables mediante el coeficiente estadístico chi-cuadrada. La base de datos producto de la encuesta levantada se manipuló utilizando el sistema Statistical Package for the Social Sciences (spss), versión 19.

Finalmente, se procedió a explicar el comportamiento de los electores y el resultado de los comicios locales llevados a cabo el 4 de julio del 2010 en Ciudad Juárez, tomando en cuenta tanto los resultados de la encuesta en comento sobre preferencias electorales, como los postulados centrales de las diferentes teorías sobre la conducta del elector. Se decidió realizar la encuesta en Ciudad Juárez debido a que durante los años de 2008, 2009 y 2010 esta ciudad fue la zona metropolitana con mayor número de personas ejecutadas y una de las que más ha sufrido por el aumento del índice de la violencia y la inseguridad pública en México producto de la llamada "guerra contra el narcotráfico".

Para dar coherencia a nuestros argumentos dividimos este trabajo en cuatro apartados. En el primero presentamos las aproximaciones básicas sobre las teorías que explican el comportamiento electoral y su relación con los escenarios de criminalidad e inseguridad pública; en el segundo se expone el escenario social marcado por la criminalidad y la inseguridad pública que impera en Ciudad Juárez; en el tercero se presenta un análisis de los resultados arrojados por la encuesta levantada ex profeso; el cuarto apartado se presenta a modo de conclusiones e implicaciones.

\section{Inseguridad pública y comportamiento electoral: aproximaciones básicas}

A pesar de que la inseguridad pública se ha convertido en un serio problema en diferentes países, no existen estudios serios que traten de conocer con precisión el impacto de ésta en el comportamiento del elector. Sin embargo, sí existen trabajos de investigación que abordan el estudio de la violencia política y la guerra civil en el comportamiento de los votantes. Tales son los casos de los trabajos de Fernández y Aguilera (2002) y Sandoval (2008). Fernández y Aguilera (2002) señalan que la violencia 
política puede convertirse, en algún momento, en un agente persuasivo del comportamiento electoral y que la propia violencia política ya es por sí misma un fenómeno social persuasivo. Respecto de la inclinación del voto, preferencias electorales y filiaciones políticas por parte de los electores en comicios desarrollados en un contexto de alta violencia política, como el caso de Santander en Colombia, sostienen que se presenta una fuerte tendencia para favorecer a opciones políticas diferentes a las que representan e identifican a los partidos políticos tradicionales, aumentando el apoyo hacia los grupos y partidos políticos independientes.

Por su parte, Sandoval (2008) concluye que en las elecciones locales, las acciones unilaterales de la guerrilla y los combates entre el gobierno y la guerrilla son las acciones de conflicto que mayor incidencia tienen en los resultados electorales. En consecuencia, las acciones de conflicto, sean de carácter paramilitar o guerrillero en todas las elecciones analizadas en Colombia durante los años 1997 y 2006, tienden a explicar el comportamiento de los resultados electorales determinados en torno a la participación, concentración y polarización electoral.

En este mismo sentido se encuentra el estudio de Blais (2006), quien señala que los aspectos psicológicos, relacionados con el entorno que percibe el votante del medio en el cual habita y se desenvuelve socialmente, inciden en la decisión personal y colectiva del voto a partir de la percepción de riesgo en la decisión de participación social.

Sobre el estudio de los efectos que las campañas electorales generan en la conducta de los votantes (independientemente del contexto en el que se desarrollan) existe una amplia bibliografía, ya que en los últimos lustros se han consolidado diferentes teorías. Estos estudios incluyen también, los efectos que los medios de comunicación generan en los votantes por la "agenda informativa" que privilegian, así como por el tratamiento mediático que hacen estos medios de los asuntos que conciernen a una determinada audiencia.

Por ejemplo, la teoría de los efectos, impulsada por lo que se conoció como la Escuela de Sociología de Chicago en los años cuarenta del siglo pasado, sostuvo que los medios de comunicación ejercen un fuerte poder sobre las ideas de las personas y, en lo particular, que durante los procesos electorales existe una influencia directa de la comunicación política en la conducta de los votantes, de tal manera que el resultado de los comicios 
electorales es determinado o afectado por el tipo y carácter de la campaña (Mendelsohn y O'Keefe, 1976; Noélle-Neumann, 1983). ${ }^{2}$ Es decir, más que el contexto en el que se desarrolla la elección, en un sistema de cuño democrático, la comunicación política en las campañas electorales es determinante y define el carácter de la representación pública, de tal forma que las preferencias de los votantes se rigen por las circunstancias de cada elección (Patterson, 1980; Iyengar, Peters y Kinder, 1982; Page, Shapiro y Dempsey, 1987; Bartels, 1988; Fan, 1988). De esta manera, de acuerdo con esta concepción, las preferencias electorales de los votantes siempre pueden ser modificadas por las campañas y en lo particular por la comunicación política (Graber, 1980; Campbell et al., 1992; Geer, 1988; Norris et al., 1999).

Sin embargo, existen otras apreciaciones teóricas que contradicen los supuestos de la teoría de los efectos, como es el caso de la teoría conocida como de la Universidad de Columbia, la cual postula que las campañas no son determinantes para el resultado final de los comicios, cumpliendo solamente un papel de reforzamiento de predisposiciones electorales generadas por una previa identidad partidista, social e ideológica (Lazarfeld, Berelson y Gaudet, 1944; McCombs y Shaw, 1972; Butler y Kavanagh, 1997). En este sentido, las campañas electorales son importantes sólo porque activan y refuerzan predisposiciones latentes existentes entre los votantes, lo cual no resulta en la ganancia de nuevos adherentes, sino más bien, ayudan a la prevención de la pérdida de los votantes ya inclinados o anclados favorablemente, generando efectos mínimos sobre la conducta del elector (Heath et al., 1991).

En este mismo sentido, Kappler (1960) señala que los medios de comunicación de masas no son la causa habitual de los cambios en el comportamiento o las actitudes de los electores, sino más bien sirven para reforzar, a través de la exposición, percepción y retención selectiva, las disposiciones preexistentes. Los medios de comunicación operan en el seno de un

\footnotetext{
${ }^{2} \mathrm{Al}$ respecto, en una encuesta realizada por el ceo de la Universidad de Guadalajara a 300 habitantes de la zona metropolitana de Guadalajara sobre los efectos de los medios en la construcción de opinión pública, $48.3 \%$ de los entrevistados consideró que los personajes públicos pueden influenciar a la sociedad con lo que comunican a nivel masivo; 33.7\% señaló que sí, pero sólo influyen en algunas personas; $11.6 \%$ afirmó que no y $6.4 \%$ dijo que no sabía o que no le interesaba (Mendoza, 2006).
} 
grupo de influencias, como la familia, la religión, los amigos, la escuela, etc., los cuales son más importantes en la creación de actitudes, creencias y comportamientos (Cooper y Jahoda, 1947).

Por su parte, la teoría económica del voto, también conocida como Rational Choice, que por cierto difiere igualmente de los postulados de la teoría de los efectos, apunta que las utilidades esperadas por los votantes de los resultantes de su acción política generan preferencias sobre los diversos cursos de acción de los ciudadanos (Downs, 1957). Los electores prefieren a los candidatos y partidos que les generan una real o perceptivamente mayor utilidad (Kreps, 1990). El ciudadano reconoce su propio interés y evalúa a todos los candidatos y partidos según sus intereses personales, votando por el que mejor valora (Enelow y Hinich, 1984). En este sentido, las campañas no generan efectos persuasivos mayores ya que el resultado electoral puede predecirse en función de unos pocos indicadores económicos (Sanders, 1997).

En los últimos años han surgido nuevas concepciones teóricas y estudios empíricos que recalcan la importancia creciente de los medios de comunicación y en lo particular de la televisión en la conducta del elector en un contexto caracterizado por la agudización de la crisis de credibilidad de la política e identidad de la gente con los partidos y la extenuación de las ideologías, así como por el papel crecientemente protagónico que tienen en las campañas la personalidad, la imagen y el carisma de los candidatos (Campbell et al., 1992; Geer, 1998; Norris et al., 1999). Es decir, estas nuevas concepciones, como por ejemplo, la teoría de los efectos cognitivos, ${ }^{3}$ la agenda setting ${ }^{4}$ y la de persuasión política, apuntan que las campañas sí influencian la conducta de los votantes (Patterson, 1980).

\footnotetext{
${ }^{3}$ Esta teoría señala que los individuos tratan de mantener sus actitudes, creencias y comportamientos de acuerdo entre sí, prestando atención a los mensajes que estén en consonancia con sus opiniones previas, buscando información que refuerce su decisión, por ejemplo, prestando atención a los espots de campaña del candidato a favor del cual se ha decidido, protegiéndose, a su vez, de información que cuestione su decisión (Festinger, 1957). El individuo puede distorsionar, deformar, interpretar incorrectamente o argumentar en contra de la información disonante que apoya al candidato por el que no se ha decidido.

${ }^{4}$ MaCombs y Shaw (1972), creadores de esta teoría, señalan que los medios de comunicación consiguen transferir al público la importancia que otorgan a los temas que se discuten en campaña y de esta manera los candidatos y partidos influyen en los votantes.
} 
Todos estos últimos estudios sobre el efecto de las campañas, los medios y la comunicación política en la conducta del elector, así como las investigaciones que abordan el análisis de la violencia política en su relación con el comportamiento del votante, aportan luces para el mejor entendimiento de la conducta del elector en un contexto de severa crisis de seguridad pública. Sin embargo, no existen estudios serios que hablen, en lo particular, sobre los efectos que genera la crisis de seguridad pública en la conducta de los electores.

Los distintos trabajos de investigación desarrollados en Estados Unidos, países europeos y algunos países latinoamericanos como Colombia, El Salvador y Nicaragua, se han enfocado al papel que han tenido las campañas, los medios de comunicación, la violencia política y la guerra civil en la conducta del electorado, pero no han particularizado en indagar sobre el efecto que genera en lo particular la crisis de seguridad pública en el comportamiento electoral.

\section{Inseguridad, miedo y malestar entre los electores}

La inseguridad pública, manifestada en el aumento de los niveles de criminalidad, genera la movilización de dos emociones básicas del ser humano: miedo y malestar. Miedo a ser víctima de los actos delictivos y malestar social porque es una responsabilidad del Estado proporcionar seguridad a sus habitantes.

Estas dos emociones se han usado como instrumentos de control, dominación, persuasión y movilización política por parte de las élites y grupos gobernantes desde épocas inmemorables.

Primero fue el miedo a la furia de los dioses y a la ira de la naturaleza, así como a las pestes por las destrucciones y cataclismos generados. Después fue el miedo a las guerras producidas por la disputa de los imperios por territorios y riquezas, ante los efectos devastadores que generaban. En tiempos más modernos, bajo regímenes autoritarios y totalitarios, fue el miedo al comunismo, al militarismo y a los gobernantes tiranos, que se legitimaban en el poder por el uso de la fuerza y la instauración de una política del terror hacia sus opositores. Hoy día, bajo sistemas democráticos, es el miedo a la criminalidad y a la violencia, a la debacle económica, a la pobreza, a los radicalismos y al terrorismo, entre otros fenómenos. 
El miedo se ha constituido como política de Estado y como instrumento de control y dominación, generando pueblos atemorizados, indignados y fastidiados (Bauman, 2007; Robin, 2004). La realidad con más violencia y criminalidad, aumenta más el miedo ciudadano ante la impotencia y la frustración que esta grave situación genera.

La construcción y el ejercicio del poder político se sustentan, en parte, con base en la movilización de las emociones y sentimientos del ser humano. Ya no se apela a la razón, sino al sentimiento y la emoción de la gente. En esta estratagema, el miedo, verdugo de la creatividad y la libertad social, se ha instituido como un instrumento paradigmático de la política, usado por igual bajo regímenes tanto autoritarios y totalitarios como democráticos, ya que el miedo es un instrumento ejemplar de represión tanto a nivel público como individual (Robin, 2004).

El miedo como instrumento de la política ha sido estudiado por diferentes teóricos del poder. Thomas Hobbes (1987) en su clásico El Leviatán consideraba que la sociedad está fundada sobre el miedo y que sin miedo no habría política. ${ }^{5}$ De hecho, Hobbes consideraba que la política es una respuesta al miedo. Por su parte, Maquiavelo en el siglo xvi consideraba que el miedo es un determinante sustancial del comportamiento del ser humano. De ahí que haya aconsejado que es más importante ser temido que ser amado.

Marco Tulio Cicerón consideraba que todo mundo se mantiene en un estado de miedo constante y que el hombre moldea su comportamiento ya sea por la ignominia, la esperanza o por el miedo. Jean Paul Sartre señalaba que el hombre es a la vez miedo y angustia. Finalmente, el ministro de propaganda de Adolfo Hitler, Joseph Goebbels, solía decir "que muchos tienen un precio y los otros miedo", entronizando el soborno y el terror como política de persuasión.

Sobre la ira, el filósofo francés Michel Foucault (1976) consideraba que ésta era algo esencialmente humano. Maquiavelo aconseja, por su parte, que todo príncipe debería cuidarse de no desbordar la ira del pueblo. Finalmente, Goebbels señalaba que en un afán de lograr la persuasión era necesario apelar en algunos casos al amor, y en otros al miedo, la ira, la esperanza o la culpa.

\footnotetext{
${ }^{5}$ Hobbes señalaba que "el miedo que disuade a los hombres de obrar mal no procede del hecho de que se establezcan castigos, sino de que se cumplan. Porque valoramos el futuro por el pasado, y rara vez esperamos lo que rara vez suele suceder".
} 
Ahora bien, las campañas electorales exitosas articulan sus estrategias no sólo con base en sus propuestas, ideas y proyectos de nación, sino también tomando en consideración la movilización de las emociones (principalmente la ira y el miedo) de los electores, generadas, entre otras razones, por la inseguridad pública. Por un lado, miedo a perder la vida, a perder la tranquilidad, a que se pierda el patrimonio que se tiene o se atente en contra de la paz y la estabilidad social. Es decir, la estrategia electoral se centra en culpar a los opositores, ya sea de ser los causantes de la crisis de inseguridad o de ser incompetentes para solucionar este problema, por lo que se publicitan como la mejor alternativa para poder enfrentar de manera exitosa a los criminales. Por el otro, apelando a la inconformidad, molestia, frustración, enojo e ira de los electores por la situación de incertidumbre y zozobra prevaleciente.

Apelar al miedo que genera la criminalidad es propio, aunque no exclusivo, de campañas electorales de partidos en el poder ya que generalmente se dice que si llegan los opositores se correrán más riesgos o las cosas pueden empeorar. Apelar a la ira de los votantes, lo hacen generalmente los partidos y candidatos que están en la oposición, acusando a los gobernantes de ser incapaces de solucionar el problema de la inseguridad y de combatir frontalmente a los criminales.

\section{Ciudad Juárez 2008-2010: crisis de inseguridad pública y procesos electorales}

El arribo del año 2008 dio pie a la ola de violencia más importante en la historia de la localidad. Así, hechos violentos antes poco comunes, como los asesinatos entre miembros de bandas criminales rivales en sitios públicos y concurridos, incendio de negocios de manera intencional, robo de automóviles con violencia o carjacking, extorsiones, ${ }^{6}$ secuestros $^{7}$ y "venta

\footnotetext{
${ }^{6}$ La violencia también implica costos económicos. Cifras proporcionadas por la Confederación Patronal de la República Mexicana (Coparmex) muestran que a partir de enero del 2008 han cerrado en la localidad alrededor de 10 mil establecimientos de distinto giro comercial.

${ }^{7}$ Durante el 2009 fueron denunciados en la ciudad 150 secuestros, lo que equivale a una tasa de poco más de cien plagios por millón de habitantes, mientras que Vene-
} 
de seguridad" por parte de las mismas células delictivas, se convirtieron en parte de la cotidianidad fronteriza.

Los asesinatos tipo ejecución se presentan como la expresión más violenta de la crisis de inseguridad pública y deterioro social en Ciudad Juárez. La forma de operar por parte de las células del crimen organizado en la localidad se caracteriza por su extrema crueldad que multiplica el sufrimiento humano, así como la no distinción entre hombres, mujeres, niños, adultos mayores y mujeres embarazadas. Las ejecuciones han alcanzado a miembros de las distintas esferas: empresarios, estudiantes y docentes universitarios, políticos, periodistas, estudiantes preparatorianos, futbolistas profesionales, líderes sociales; es decir, todas las esferas sociales han sido trastocadas por la violencia. Las cifras oficiales muestran la realidad juarense: a partir del inicio de la ola de violencia en enero del 2008, el número de ejecuciones se incrementó de manera alarmante: durante el 2008 la cifra ascendió a 1,607 eventos, para el año 2009 se multiplicó a 2,601 asesinatos, y hasta el 31 de julio del 2010, los asesinatos sumaban 1,764. Cifras alarmantes, si se comparan con el total de homicidios en todo el año 2007, que fueron 301.

En este sentido, una de las ciudades de México que más ha sufrido por el aumento del índice de la violencia y la inseguridad pública ha sido Ciudad Juárez, localizada en el estado de Chihuahua, vecina de El Paso, Texas, a tal grado de ser catalogada por segundo año consecutivo como "la ciudad más violenta del mundo". Durante el año 2009 Ciudad Juárez presentó una tasa de 191 homicidios dolosos por cada 100 mil habitantes. Marcadamente distanciadas se encuentran otras ciudades que conforman a las 10 ciudades más violentas del mundo: en segundo lugar figura San Pedro Sula, Honduras, con una tasa de 119; San Salvador, capital de El Salvador, con una tasa de 95; Caracas, Venezuela, con 94; Guatemala, Guatemala, 86; Cali, Colombia, 76; Tegucigalpa, Honduras, y Nueva Orléans, Estados Unidos, con 69; Medellín, Colombia, 68; y Cape Town, Sudáfrica, con 68. Medellín es la urbe que hasta ahora ha alcanzado las tasas

zuela, el líder mundial en este delito, en 2009 tuvo una tasa de 15 secuestros por cada millón de habitantes, es decir, seis veces menos que Ciudad Juárez. En Colombia, el peor año de secuestros fue 1999, con una tasa de 64 secuestros por millón de habitantes, 36\% menos que la alcanzada en Ciudad Juárez durante el 2009. 
de homicidio más elevadas en el mundo en al menos los últimos 30 años. A inicios de los años noventa llegó al máximo de 250 homicidios dolosos por cada 100 mil habitantes (Seguridad, Justicia y Paz, 2010).

El aumento sustantivo de la criminalidad ha hecho que los habitantes de esta metrópoli alteren su estilo de vida ya que la inseguridad ha propiciado la virtual cancelación de las prácticas habituales de convivencia social, principalmente las que se realizan en espacios públicos, independientemente de los daños psicológicos y sociales para los juarenses.

Debido a la condición de ciudad fronteriza, en la actualidad se presenta una interesante dinámica de autoexilio de juarenses a otros lugares de México y de Estados Unidos, especialmente a la ciudad vecina de El Paso, Texas. El Observatorio de Seguridad y Convivencia Ciudadana de la Universidad Autónoma de Ciudad Juárez, mediante la encuesta de percepción ciudadana sobre inseguridad (2010), reporta que durante los años de 2008, 2009 y 2010230 mil habitantes abandonaron la ciudad por causas económicas, de inseguridad o ambas. Esta cifra representa casi una quinta parte del total de habitantes. El mismo estudio señala que el destino del $54 \%$ de los habitantes que abandonaron la ciudad fue Estados Unidos, el resto se dirigió al interior del estado de Chihuahua o a otras entidades federativas de la República.

El aumento de la criminalidad, la violencia y la inseguridad pública ha generado efectos devastadores en materia económica, institucional y turística. Esta ola criminal expone el deterioro de las instituciones del Estado en sus tres niveles, la impertinencia de los programas emergentes para enfrentar a las organizaciones criminales y la ineficiencia del marco jurídico y legal vigente en el estado de Chihuahua. Otro de los efectos negativos es la concentración de los recursos públicos en la lucha armada contra el narcotráfico con la respectiva reducción del presupuesto para la obra pública y programas de desarrollo social. La ineficiencia de los esfuerzos oficiales por contener los índices delictivos genera una alta percepción social de elevados niveles de corrupción entre los responsables de garantizar el bien común.

La estrategia gubernamental de confrontación armada contra el narcotráfico ha propiciado que el Estado se coloque en medio de dos actores, la sociedad y el crimen organizado. Debido a esta coyuntura, el Estado dirige un discurso en forma de declaraciones políticas distinto para cada actor (Paniagua, 2009). 
Por una parte, hay un discurso para dirigirse a la sociedad, un discurso ideológico que se encamina a la justificación de su accionar, intentando siempre que se acepten los recursos públicos ejercidos como parte de la guerra contra el narcotráfico, en especial el mediático Operativo Conjunto Chihuahua, puesto en marcha el 27 de marzo de 2008. La principal idea es hacer creer que todo va de acuerdo con lo planeado, que los resultados son positivos. La propaganda oficial se basa en la costumbre de señalar al narcotráfico y sus derivados en términos moralistas, en lugar de implementar innovaciones políticas capaces de disminuir el impacto social negativo; la propaganda moralista es una alternativa oficial para lograr su aprobación por parte de la sociedad.

De acuerdo al tiempo transcurrido, al derroche de recursos, a la falta de resultados y a la exposición de la ineficacia gubernamental por reducir el impacto social de la "guerra contra el narcotráfico", el discurso oficial dirigido a la sociedad ha cambiado, y ahora parece existir una estrategia pública a todos los niveles por la criminalización a priori de las víctimas, un intento por justificar los homicidios de seres humanos inocentes, es decir, una especie de "el narco somos todos".

Por otra parte, hay un discurso dirigido a las células del crimen organizado. Éste forma parte de la estrategia oficial por concretar el dominio sobre los criminales y lograr el control del escenario violento.

Parte de esta estrategia es demostrar, vía discurso, el monopolio de los recursos legales que el Estado ha implementado y la posible contundencia del uso de las fuerzas armadas.

En conjunto, estos elementos propician una drástica caída de la imagen pública de Ciudad Juárez y del estado de Chihuahua al interior de México y ante la sociedad internacional. Ante críticos y especialistas se gesta la idea de un Estado-nación mexicano "fallido". ${ }^{8}$

\footnotetext{
${ }^{8}$ La percepción generalizada de que en México las políticas encaminadas a contener al crimen organizado no generan los resultados esperados, ha dado pie a debatir sobre el "Estado fallido". La revista estadounidense Foreign Policy elabora y publica el índice de Estados fallidos, que abarca las esferas social, económica y política y analiza 12 indicadores. En el ámbito social se miden las tendencias demográficas, el movimiento de refugiados, el revanchismo de ciertos grupos y la recurrencia del conflicto o enfrentamiento. En el terreno económico, los índices enfatizan el desarrollo desigual y el declive/estancamiento. Finalmente, en lo político destacan la criminalidad y la pérdida
} 
Ahora bien, el 4 de julio del 2010 se realizaron los comicios locales para elegir al presidente municipal, al síndico de esta ciudad, así como para seleccionar a los titulares de siete escaños en el Congreso local correspondientes al municipio de Juárez y el gobernador del estado de Chihuahua.

Los partidos políticos y sus candidatos contendientes por la presidencia municipal de Ciudad Juárez fueron: César Jáuregui Moreno, Partido Acción Nacional (PAN); Víctor Manuel Reyes Gloria, Partido de la Revolución Democrática (PRD); Lluvia Luna Nevárez, Convergencia; y Héctor Agustín Murguía Lardizábal, candidatura común conformada por el Partido Revolucionario Institucional (PRI), el Partido Verde Ecologista de México (PVEM) y el Partido Nueva Alianza (PANAL); el Partido del Trabajo (PT) no registró candidato a la presidencia municipal de Ciudad Juárez.

Por su parte, los partidos políticos y sus candidatos a la sindicatura municipal fueron: Karla Uraga Peralta, PAN; Miguel Dávila Parada, PRD; Arturo Hernández García, Convergencia; Brenda Araceli Hernández Martínez, PT; y José Luis Canales de la Vega, candidatura común conformada por el PRI, el PVEM y el PANAL.

Mientras que por la gubernatura del estado de Chihuahua los partidos políticos y sus candidatos fueron: Carlos Borruel Vaquera, PAN; Luis Adolfo Orozco Orozco, PRD; César Horacio Duarte Jàquez, candidatura común conformada por el PRI, el PVEM, el PT y el PANAL. Convergencia no registró candidato a la gubernatura del estado.

Como se menciona líneas arriba, para el caso del municipio de Juárez, adicionalmente estuvieron en juego las diputaciones adscritas a los distritos electorales locales II, III, IV, V, VI, VII y VIII.

El resultado final del proceso electoral estatal 2010 en Ciudad Juárez sobre el cómputo de votos destinados a los candidatos para la gubernatura del estado fue el siguiente: César Horacio Duarte Jáquez, candidatura común conformada por el PRI, el PVEM y el PANAL, alcanzó 166,530 votos

de legitimidad de las autoridades, el deterioro progresivo de los servicios públicos, las violaciones de los derechos humanos, el aparato de seguridad del Estado y su uso, el auge de ciertas élites/facciones y la intervención de otros Estados o actores externos. Por su parte, el estadounidense Noam Chomsky (2006) señala que un Estado fallido es el que no logra suministrar seguridad a su población, ni le garantiza derechos dentro y fuera del país y no mantiene el funcionamiento (no meramente formal) de las instituciones democráticas. 
o $53.31 \%$ del total de sufragios efectivos, la participación fue de $29.59 \%$. La cantidad de votos logrados a favor de la candidatura común en Juárez fue $27.73 \%$ del total estatal. La participación estatal promedio para elegir gobernador fue de $41.41 \%$. En segundo lugar finalizó Carlos Borruel Vaquera, candidato del PAN, con 127,809 sufragios a favor o $40.91 \%$ del total de votos efectivos.

En lo que a la presidencia municipal se refiere, los resultados finales fueron: Héctor Agustín Murguía Lardizábal, candidatura común conformada por el PRI, el PVEM y el PANAL, alcanzó 162,146 votos o $52.26 \%$ del total de sufragios efectivos, la participación fue del 29.39\%, muy por debajo del promedio estatal para este cargo que fue de 41.06\%. César Jáuregui Moreno, candidato del PAN, terminó en segundo lugar con 118,287 sufragios a favor o $38.12 \%$ del total de votos efectivos.

Mientras que la carrera electoral por la sindicatura reflejó los siguientes datos: José Luis Canales de la Vega, candidatura común conformada por el PRI, el PVEM y el PANAL, alcanzó 152,740 votos o $49.21 \%$ del total de sufragios, la participación fue del $29.40 \%$, muy por debajo del promedio estatal que fue de $41.05 \%$; Karla Uraga Peralta, candidata del Pan, finalizó en segundo lugar con 119,430 votos o $38.47 \%$ de sufragios efectivos.

Adicionalmente, en el municipio de Juárez también se registraron procesos para elegir diputados locales; los resultados reflejaron el siguiente mapa electoral: los candidatos propuestos por el PRI ${ }^{9}$ se agenciaron victorias en los distritos II, V, VI, VII y VIII, mientras que el PAN y sus candidatos triunfaron en las demarcaciones III y IV.

Este periodo electoral local 2010 venía precedido por los procesos electorales locales del $2004^{10}$ y $2007,{ }^{11}$ y por los comicios federales del

\footnotetext{
${ }^{9}$ A diferencia de las candidaturas para la gubernatura estatal, la presidencia y el síndico municipal, el PRI no formó candidaturas comunes con otros partidos en ningún distrito.

${ }^{10}$ El proceso electoral local del 2004 arrojó como resultado que los candidatos emanados de la Alianza con la Gente (PRI, PVEM, PT) a la gubernatura del estado, José Reyes Baeza Terrazas (993,511 votos o $42.19 \%$ de los votos efectivos; $44.20 \%$ de participación); la presidencia municipal, Héctor Agustín Murguía Lardizábal (181,604 votos o $54.72 \%$ de los votos efectivos; $36.53 \%$ de participación); y el síndico municipal, Wilfrido Otto Guillermo Campbell Saavedra (175,384 votos o 52.88\% de los votos efectivos; $36.51 \%$ de participación) ocuparan estos puestos. Mientras que en las diputaciones
} 
$2006^{12}$ y 2009. ${ }^{13}$ Durante los procesos de 2009 y 2010 Ciudad Juárez enfrentó elecciones locales y federales en el contexto de la más severa crisis de inseguridad pública y criminalidad de su historia.

La inusitada ola de violencia presentada en Ciudad Juárez desde enero del 2008 coloca a la violencia y la inseguridad pública como el principal reclamo de los juarenses. En especial, se exige solucionar problemas poco comunes antes de esa fecha y de alto impacto social como secuestros, extorsiones, incendios intencionados de negocios, robo de autos con violencia o carjacking y asesinatos en todo lugar y momento. En las campañas electorales, los diversos candidatos colocaron a la inseguridad como el principal problema, aunque también se hizo énfasis en el desempleo y en la necesidad de gestionar un desarrollo social a largo plazo.

correspondientes a los siete distritos locales adscritos al municipio de Juárez los resultados arrojaron cuatro distritos (II, V, VI y VII) para la Alianza con la Gente (PRI, PVEM y PT); y dos distritos (III y IV) para la alianza Todos Somos Juárez (PAN, PRD y Convergencia) (cifras del Instituto Estatal Electoral).

${ }^{11}$ El proceso electoral local intermedio del 2007 arrojó como resultado que los candidatos emanados por la Alianza por Juárez (PRI y PANAL) a la presidencia municipal, José Reyes Ferriz (148,788 votos o $53.77 \%$ de los votos efectivos; $27.85 \%$ de participación), y el síndico municipal Eloy Leonardo Villar Calvillo (130,720 votos o 47.20 de los votos efectivos, $27.88 \%$ de participación) ocuparan esos puestos. Mientras que en las diputaciones correspondientes a los siete distritos locales adscritos al municipio de Juárez los resultados arrojaron seis distritos (II, III, V, VI, VII y VIII) para la Alianza por Juárez (PRI y PANAL); y un distrito (IV) para el PAN (cifras del Instituto Estatal Electoral).

${ }^{12}$ A diferencia de la distribución local, el municipio de Juárez cuenta sólo con cuatro distritos. El proceso electoral federal 2006 arrojó los siguientes candidatos electos: el distrito I Enrique Serrano Escobar (45,482 votos o $38.29 \%$ de los votos efectivos, $44.15 \%$ de participación); distrito II Lilia Merodio Reza (38,118 votos o $43.35 \%$ de los votos efectivos, $37.38 \%$ de participación); distrito IV Víctor Valencia de los Santos (38,897 votos o $37.97 \%$ de los votos efectivos, $42.81 \%$ de participación), candidatos de la Alianza por México (PRI y PVEm de México); por su parte, el distrito III lo ganó Cruz Pérez Cuéllar del pan (64,827 votos o $51.12 \%$ de los votos efectivos, $49.33 \%$ de participación). La participación general del municipio de Juárez reflejó 43.52\%.

${ }^{13}$ El proceso intermedio electoral federal 2006 arrojó los siguientes candidatos electos: el distrito I Jaime Flores Castañeda (30,625 votos o $33.62 \%$ de los votos efectivos, 44.15\%); distrito II Héctor Agustín Murguía Lardizábal (29,347 votos o 48.08\% de los votos efectivos); distrito IV Adriana Terrazas Porras (23,844 votos o $30.06 \%$ de los votos efectivos), candidatos del PRI; por su parte, el distrito III lo ganó María Antonieta Pérez del Pan (24,174 votos o $30.96 \%$ de los votos efectivos). 
A través del proceso electoral de 2010, la ciudadanía fue testigo de las valoraciones, prejuicios y conductas que impidieron el diálogo y la competencia democrática; de esta manera, diarios, revistas, portales de Internet y correos anónimos dieron cabida a "expresiones negras" cuyo objetivo central fue sin duda la descalificación del contrario. El punto culminante de la también llamada "guerra sucia" se llevó a cabo durante el debate entre candidatos a la presidencia municipal convocado y organizado por el Instituto Estatal Electoral. Durante el desarrollo del mismo, César Jáuregui Moreno, candidato del PAN, acusó las supuestas relaciones comerciales del candidato común del PRI, PVEM y PANAL, Héctor Agustín Murguía Lardizábal, con Baldomero Fuentes, lugarteniente de Vicente Carrillo, líder de la organización criminal conocida como el Cártel de Juárez.

Cabe señalar que durante el proceso electoral Murguía fue constantemente acusado de sus supuestos nexos con grupos de narcotraficantes; sin embargo, a la fecha tales acusaciones no han sido aclaradas ni sustentadas -más allá, no se sabe si existe algún proceso judicial encaminado a aclarar los supuestos nexos.

Los partidos políticos, sus militantes y simpatizantes utilizaron de manera sistemática las redes sociales como facebook, twitter y youtube para penetrar más efectivamente en el electorado, pero con mensajes, contenidos y posturas de precario valor. Por medio de las herramientas virtuales se tejió una confrontación directa plagada de ataques personales, acusaciones mutuas de corrupción, ineficacia, deshonestidad y calumnias cuyo único objetivo fue desprestigiar al oponente y, en su caso, atraer a causa propia el máximo de votos posibles.

\section{Análisis de los resultados}

Del estudio de campo se desprende una alta insatisfacción por parte de la sociedad juarense respecto del funcionamiento de los gobernantes, candidatos y partidos políticos, que no han podido brindar la seguridad que por ley están obligados a proporcionar a los ciudadanos.

Esta inseguridad pública generó un costo político para todos los partidos y candidatos que compitieron en la elección local del 4 de julio de 2010, principalmente reflejado en el alto nivel de abstencionismo que alcanzó cifras importantes: en la contienda para elegir gobernador se abs- 
tuvo $70.41 \%$; mientras que para elegir presidente municipal la cifra de abstencionistas alcanzó $70.73 \%$ de la lista nominal (IEe, 2010). ${ }^{14} \mathrm{Si}$ bien el abstencionismo en la ciudad es un fenómeno permanente en el pasado reciente (Padilla, 2010), en esta elección la inseguridad pública se presentó como un elemento coyuntural.

Un análisis de correlación entre las variables "La situación de inseguridad pública que sufre la ciudad será determinante para orientar mi voto el próximo 4 de julio” y “¿Votará en estas próximas elecciones?” muestra que $73 \%$ de los encuestados que están "muy de acuerdo" en que la inseguridad pública determina su voto no pensaba votar; el porcentaje para los que estaban "algo de acuerdo" es de 80.2 , mientras que $70 \%$ estuvo "de acuerdo". En contraparte, aquellos que opinaron "muy de acuerdo" en que la inseguridad pública determina su voto, sólo $16.8 \%$ pensaba votar; el porcentaje para los que estaban "algo de acuerdo" bajó al 3.5\%; mientras que $12.9 \%$ que estuvo "de acuerdo" pensó en acudir a votar.

Si bien la crisis de inseguridad pública que azota Ciudad Juárez influye de manera negativa en la decisión de votar, el costo mayor no necesariamente fue para el partido gobernante (PRI) a nivel estatal y local sino para el partido que tiene la responsabilidad del gobierno federal (PAN). ¿’Por qué ante una crisis de inseguridad pública que provoca insatisfacción ciudadana el partido en el gobierno municipal y estatal gana las elecciones?

El triunfo del PRI y la candidatura común que encabezó se debió, entre otras cosas, a que entre el electorado se tiene la percepción de que el PRI y sus candidatos están más capacitados para enfrentar el problema de seguridad pública que el resto de los partidos y que relativamente ha dado mejores resultados que otros sobre la materia.

$\mathrm{Al}$ respecto, el cruce de las variables "El partido que está más capacitado para enfrentar el problema de la inseguridad pública es...” y "¿Votará en estas próximas elecciones?” arrojó los siguientes resultados: El 10.7\% de aquellos que contestaron que el PRI ${ }^{15}$ era el partido más capacitado para

\footnotetext{
${ }^{14}$ En la elección de síndico municipal el abstencionismo alcanzó 70.6\%; para elegir diputado por el distrito II 72.88\%; distrito III 70.3\%; distrito IV 67.05\%; distrito V $71.74 \%$; distrito VI $69.33 \%$; distrito VII 70.71\%; y distrito VIII $71.88 \%$ (IEE, 2010).

${ }^{15} \mathrm{Al}$ respecto, el PVEM obtuvo $7.9 \%$ en este cruce -hay que recordar que días después de concluido el proceso de recolección de datos para este trabajo, se formalizó la figura de candidatura común válida para el PRI, PANAL y el PVEM.
} 


\section{Cuadro 1}

Tabulación cruzada entre las variables "La situación de inseguridad pública que sufre la ciudad será determinante para orientar mi voto el próximo 4 de julio" y “¿Votará en estas próximas elecciones?”

\begin{tabular}{|c|l|c|c|c|c|c|}
\hline \multicolumn{2}{|c|}{} & \multicolumn{5}{|c|}{$\begin{array}{c}\text { ¿La situación de inseguridad pública que sufre la ciudad será } \\
\text { determinante para orientar mi voto el próximo 4 de julio? }\end{array}$} \\
\cline { 2 - 7 } & $\begin{array}{c}\text { Muy de } \\
\text { acuerdo }\end{array}$ & $\begin{array}{c}\text { Algo de } \\
\text { acuerdo }\end{array}$ & $\begin{array}{c}\text { De } \\
\text { acuerdo }\end{array}$ & $\begin{array}{c}\text { En } \\
\text { desacuerdo }\end{array}$ & $\begin{array}{c}\text { Completamente } \\
\text { en desacuerdo }\end{array}$ \\
\hline \multirow{2}{*}{$\begin{array}{c}\text { ¿Votará en } \\
\text { estas próximas } \\
\text { elecciones? }\end{array}$} & No & $73.0 \%$ & $80.2 \%$ & $70.0 \%$ & $52.6 \%$ & $36.8 \%$ \\
\cline { 2 - 7 } & Sí & $16.8 \%$ & $3.5 \%$ & $12.9 \%$ & $31.6 \%$ & $47.4 \%$ \\
\cline { 2 - 7 } & $\begin{array}{c}\text { No sabe / } \\
\text { No contestó }\end{array}$ & $10.3 \%$ & $16.3 \%$ & $17.1 \%$ & $15.8 \%$ & $15.8 \%$ \\
\hline
\end{tabular}

* El valor de chi-cuadrada es de 32.34 con 8 grados de libertad al nivel 0.000 .

Fuente: Elaboración propia con datos de la encuesta.

enfrentar el problema de la inseguridad pública pensaba ir a votar; el porcentaje disminuye al $6.1 \%$ en el caso del PAN y al $4.1 \%$ en el PRD. Como una constante en este ejercicio se encuentra la intención de no votar. El 59\% de los encuestados que opinó que el PRD era el partido más capacitado para enfrentar el problema de la inseguridad pública no pensaba en sufragar; mientras que para el PAN este porcentaje fue de 77.6 y para el PRI de 76.0. ${ }^{16}$

En lo que a candidatos se refiere, $10.7 \%$ de quienes contestaron que los candidatos del PRI ${ }^{17}$ tienen el mejor programa contra la inseguridad pública pensaban en votar, un porcentaje mayor que los candidatos del PAN $(9.8 \%)$ y PRD $(5.1 \%)$. La intención por no votar es mayor en todos los casos, $81.4 \%$ de los encuestados que contestaron que los candidatos del PRI ${ }^{18}$ contaban con el mejor programa contra la inseguridad no iría a votar; el porcentaje para el PAN fue de 77.9 y para el PRD de 62.4. En este ejercicio de correlación sobresale la afirmación de los encuestados de que ningún partido cuenta con el mejor programa para afrontar la problemática, de éstos, $22.8 \%$ votaría y $60.8 \%$ no tenía contemplado hacerlo.

\footnotetext{
${ }^{16}$ El PVEM alcanzó $68.1 \%$.

${ }^{17}$ El PVEM obtuvo $9.7 \%$.

${ }^{18}$ El PVEM obtuvo $62.5 \%$.
} 
Cuadro 2

Tabulación cruzada entre las variables "El partido que está más capacitado para enfrentar el problema de la inseguridad pública" y “¿Votará en las próximas elecciones?”

\begin{tabular}{|c|l|c|c|c|c|c|c|}
\hline \multirow{2}{*}{} & \multicolumn{5}{|c|}{$\begin{array}{r}\text { El partido que está más capacitado para enfrentar el } \\
\text { problema de la inseguridad pública es... }\end{array}$} \\
\cline { 2 - 8 } & PRD & PAN & PRI & PVEM & Otros & Ninguno \\
\hline \multirow{2}{*}{$\begin{array}{c}\text { ¿Votará en } \\
\text { estas próximas } \\
\text { elecciones? }\end{array}$} & No & $59.4 \%$ & $77.6 \%$ & $76.0 \%$ & $68.1 \%$ & $62.5 \%$ & $62.5 \%$ \\
\cline { 2 - 8 } & Sí & $4.1 \%$ & $6.1 \%$ & $10.7 \%$ & $7.9 \%$ & $3.5 \%$ & $23.1 \%$ \\
\cline { 2 - 8 } & No sabe / & $36.5 . \%$ & $16.3 \%$ & $13.3 \%$ & $24.0 \%$ & $34.0 \%$ & $14.4 \%$ \\
\hline
\end{tabular}

*. El valor de chi-cuadrada es de 25.41 con 10 grados de libertad al nivel 0.005 .

Fuente: Elaboración propia con datos de la encuesta.

En cuanto a la correlación de las variables "Percepción de los resultados sobre seguridad pública generados por el partido gobernante (PRI) a nivel estatal y municipal” y "¿Votará en estas próximas elecciones?" los resultados muestran un escenario favorable para el partido gobernante en el estado y municipio. El $16.3 \%$ de los encuestados cuya percepción fue "sí ha dado resultados" tenía planeado ejercer su derecho a votar; por el

\section{Cuadro 3}

Tabulación cruzada entre las variables "Los candidatos que tienen el mejor programa para resolver el problema de inseguridad pública son" y “¿Votará en las próximas elecciones?"

\begin{tabular}{|c|l|c|c|c|c|c|c|}
\hline \multicolumn{2}{|c|}{} & \multicolumn{6}{|c|}{$\begin{array}{c}\text { Los candidatos que tienen el mejor programa para resolver } \\
\text { el problema de inseguridad pública son... }\end{array}$} \\
\cline { 3 - 8 } & PRD & PAN & PRI & PVEM & Otros & Ninguno \\
\hline \multirow{2}{*}{$\begin{array}{c}\text { ¿Votará en } \\
\text { estas próximas } \\
\text { elecciones? }\end{array}$} & No & $62.4 \%$ & $77.9 \%$ & $81.4 \%$ & $62.5 \%$ & $79.7 \%$ & $60.8 \%$ \\
\cline { 2 - 8 } & Sí & $5.1 \%$ & $9.8 \%$ & $10.7 \%$ & $9.7 \%$ & $14.3 \%$ & $22.8 \%$ \\
\cline { 2 - 8 } & $\begin{array}{c}\text { No sabe / } \\
\text { No contestó }\end{array}$ & $32.5 \%$ & $12.3 \%$ & $7.9 \%$ & $27.8 \%$ & $6.0 \%$ & $16.4 \%$ \\
\hline
\end{tabular}

*. El valor de chi-cuadrada es de 23.47 con 10 grados de libertad al nivel 0.009. Fuente: Elaboración propia con datos de la encuesta. 
contrario, $9.9 \%$ de aquellos que opinaron "no ha dado resultados" acudiría a votar. La intención de no acudir a votar acá también prevalece: si la percepción era el PRI "sí ha dado resultados", 72.2\% no acudiría a votar; "no ha dado resultados", 77.5\%; "no sabe o no contestó", 70.3\%.

\section{Cuadro 4}

Tabulación cruzada entre las variables "Percepción de los resultados sobre seguridad pública generados por el partido gobernante (PRI) a nivel estatal y municipal” y “¿Votará en estas próximas elecciones?”

\begin{tabular}{|c|l|c|c|c|}
\hline \multicolumn{2}{|c|}{} & \multicolumn{3}{|c|}{$\begin{array}{c}\text { Percepción de los resultados sobre seguridad pública } \\
\text { generados por el partido gobernante (PRI) a nivel estatal } \\
\text { y municipal. }\end{array}$} \\
\cline { 3 - 5 } & $\begin{array}{c}\text { Sí ha dado } \\
\text { resultados }\end{array}$ & $\begin{array}{c}\text { No ha dado } \\
\text { resultados }\end{array}$ & $\begin{array}{c}\text { No sabe/No } \\
\text { contestó }\end{array}$ \\
\hline \multirow{2}{*}{$\begin{array}{c}\text { ¿Votará en } \\
\text { estas próximas } \\
\text { elecciones? }\end{array}$} & No & $72.2 \%$ & $77.5 \%$ & $70.3 \%$ \\
\cline { 2 - 5 } & Sí & $16.3 \%$ & $9.9 \%$ & $5.4 \%$ \\
\cline { 2 - 5 } & $\begin{array}{c}\text { No sabe / } \\
\text { No contestó }\end{array}$ & $11.5 \%$ & $12.6 \%$ & $24.3 \%$ \\
\hline
\end{tabular}

* El valor de chi-cuadrada es de 17.42 con 4 grados de libertad al nivel 0.002 .

Fuente: Elaboración propia con datos de la encuesta.

A raíz del Operativo Conjunto Chihuahua y de la estrategia de rescate social Todos Somos Juárez, encabezados por el gobierno federal, la ciudadanía juarense se creó altas expectativas sobre los resultados del mismo: las desagradables experiencias relacionadas a la seguridad pública después de implementado dicho operativo generó, adicionalmente a la apatía y descontento generalizado, el llamado "voto de castigo" contra el PAN. La racionalidad del "voto de castigo" se basa en la afirmación de que los partidos políticos son derrotados en procesos electorales debido a sus errores y limitaciones en la práctica de la administración pública.

La correlación de las variables "Percepción de los resultados sobre seguridad pública generados por el partido gobernante (PAN) a nivel Federal” y “¿Votará en estas próximas elecciones?” expone desventaja para el partido del presidente de la República. El 14.5\% de los encuestados cuya percepción fue "sí ha dado resultados" pensaba en votar; por el contrario, 
$19.5 \%$ de aquellos que opinaron "no ha dado resultados" acudiría a votar. La tendencia abstencionista prevalece: si la percepción era "sí ha dado resultados", 79.2\% no acudiría a votar; "no ha dado resultados", $67.1 \%$ de los encuestados no votaría; "no sabe o no contestó", $77.5 \%$.

\section{Cuadro 5}

Tabulación cruzada entre las variables "Percepción de los resultados sobre seguridad pública generados por el partido gobernante (PAN) a nivel federal” y “¿Votará en las próximas elecciones?”

\begin{tabular}{|c|c|c|c|c|}
\hline & & \multicolumn{3}{|c|}{$\begin{array}{l}\text { Percepción de los resultados sobre seguridad pública } \\
\text { generados por el partido gobernante (PAN) a nivel } \\
\text { federal }\end{array}$} \\
\hline & & $\begin{array}{l}\text { Sí ha dado } \\
\text { resultados }\end{array}$ & $\begin{array}{l}\text { No ha dado } \\
\text { resultados }\end{array}$ & $\begin{array}{l}\text { No sabe/No } \\
\text { contestó }\end{array}$ \\
\hline \multirow{3}{*}{$\begin{array}{l}\text { ¿Votará en } \\
\text { estas próximas } \\
\text { elecciones? }\end{array}$} & No & $79.2 \%$ & $67.1 \%$ & $77.5 \%$ \\
\hline & Sí & $14.5 \%$ & $19.5 \%$ & $2.5 \%$ \\
\hline & $\begin{array}{l}\text { No sabe / } \\
\text { No contestó }\end{array}$ & $6.3 \%$ & $13.4 \%$ & $20.0 \%$ \\
\hline
\end{tabular}

*. El valor de chi-cuadrada es de 9.81 con 4 grados de libertad al nivel 0.044 .

Fuente: Elaboración propia con datos de la encuesta.

El análisis de los resultados de la correlación entre las variables "Los responsables por la inseguridad pública que sufre la ciudad son..." y "Percepción de los resultados sobre seguridad pública generados por el partido gobernante (PAN) a nivel federal expone que $91 \%$ de los encuestados que afirman que los partidos políticos son responsables por la inseguridad pública en la ciudad tiene la percepción de que el PAN "no ha dado resultados" sobre la materia; el porcentaje es de $81.8 \%$ cuando los responsables son los gobernantes; los delincuentes $81.5 \%$; los ciudadanos $60 \%$; y todos, $72.3 \%$. El resultado es muy distinto cuando la percepción es que el pan "si ha dado resultados": el $4 \%$ de los encuestados que afirman que los partidos políticos son responsables por la inseguridad pública en la ciudad tienen la percepción de que el PAN sí ha dado resultados sobre la materia; el porcentaje es de $10.7 \%$ cuando los responsables son los gobernantes; los delincuentes $7.4 \%$; los ciudadanos $14.3 \%$; y todos, $17.0 \%$. 


\section{Cuadro 6}

Tabulación cruzada entre las variables "Los responsables por la inseguridad pública que sufre la ciudad son" y "Percepción de los resultados sobre seguridad pública generados por el partido gobernante (PAN) a nivel federal"

\begin{tabular}{|c|l|c|c|c|c|c|c|}
\hline \multirow{2}{*}{} & \multicolumn{3}{|c|}{ Los responsables por la inseguridad pública que sufre la ciudad son... } \\
\cline { 3 - 8 } & $\begin{array}{c}\text { Los } \\
\text { partidos } \\
\text { políticos }\end{array}$ & $\begin{array}{c}\text { Los } \\
\text { gobernantes }\end{array}$ & $\begin{array}{c}\text { Los } \\
\text { ciudadanos }\end{array}$ & $\begin{array}{c}\text { Los } \\
\text { delincuentes }\end{array}$ & Todos & $\begin{array}{c}\text { No sabe/ } \\
\text { No contestó }\end{array}$ \\
\hline $\begin{array}{c}\text { Percepción de los } \\
\text { resultados sobre } \\
\text { seguridad pública } \\
\text { generados por el } \\
\text { partido gobernante } \\
\text { PAN a nivel federal. }\end{array}$ & $\begin{array}{l}\text { Sí ha dado } \\
\text { resultados }\end{array}$ & $4.0 \%$ & $10.7 \%$ & $14.3 \%$ & $7.4 \%$ & $17.0 \%$ & $16.0 \%$ \\
\cline { 2 - 8 } & $\begin{array}{l}\text { No ha dado } \\
\text { resultados }\end{array}$ & $91.0 \%$ & $81.8 \%$ & $60.0 \%$ & $81.5 \%$ & $72.3 \%$ & $64.0 \%$ \\
\hline
\end{tabular}

* El valor de chi-cuadrada es de 21.63 con 10 grados de libertad al nivel 0.017 . Fuente: Elaboración propia con datos de la encuesta.

Los resultados de las tabulaciones cruzadas entre las variables "Los responsables por la inseguridad pública que sufre la ciudad son..." y "El partido menos capacitado para resolver el problema de inseguridad pública es..." tampoco son favorables para Acción Nacional. El 23\% de los que señalaron a los partidos políticos como responsables de la inseguridad pública en Ciudad Juárez mencionó que el PAN era el menos capacitado para resolver el problema; seguido del PRI ${ }^{19}$ con $15.4 \%$; PRD 3.8\%; y otros $7.7 \%$. Quienes optaron por los gobernantes como responsables de la inseguridad pública en Juárez, 28.7\% mencionó que el PAN carecía de capacidad para resolver el problema; por $16.4 \%$ del PRI ${ }^{20} 15.6 \%$ del PRD; y $3.3 \%$ otros.

Las tabulaciones cruzadas entre las variables de intención de votar y orientación del voto con aquellas relacionadas al miedo y/o temor de ir a votar no se incluyen en esta sección, ya que después de utilizar el estadístico chi-cuadrada se demostró la existencia de independencia entre estas

\footnotetext{
${ }^{19}$ El PVEM obtuvo $19.2 \%$.

${ }^{20}$ El PVEM obtuvo $10.7 \%$.
} 
Cuadro 7

Tabulación cruzada entre las variables "Los responsables por la inseguridad pública que sufre la ciudad son..." y "El partido menos capacitado para resolver el problema de inseguridad pública es..."

\begin{tabular}{|c|c|c|c|c|c|c|c|}
\hline & & \multicolumn{6}{|c|}{ Los responsables por la inseguridad pública que sufre la ciudad son... } \\
\hline & & $\begin{array}{l}\text { Los partidos } \\
\text { políticos }\end{array}$ & $\begin{array}{c}\text { Los } \\
\text { gobernantes }\end{array}$ & $\begin{array}{l}\text { Los } \\
\text { ciudadanos }\end{array}$ & $\begin{array}{c}\text { Los } \\
\text { delincuentes }\end{array}$ & Todos & $\begin{array}{c}\text { No sabe/ } \\
\text { No contestó }\end{array}$ \\
\hline \multirow{6}{*}{$\begin{array}{l}\text { El partido menos } \\
\text { capacitado para } \\
\text { resolver el problema } \\
\text { de inseguridad } \\
\text { pública es... }\end{array}$} & PRD & $3.8 \%$ & $15.6 \%$ & $37.1 \%$ & $7.4 \%$ & $22.0 \%$ & $.0 \%$ \\
\hline & PAN & $23.1 \%$ & $28.7 \%$ & $8.6 \%$ & $33.3 \%$ & $17.6 \%$ & $40.0 \%$ \\
\hline & PRI & $15.4 \%$ & $16.4 \%$ & $5.7 \%$ & $11.1 \%$ & $10.1 \%$ & $10.0 \%$ \\
\hline & PVEM & $19.2 \%$ & $10.7 \%$ & $17.1 \%$ & $22.2 \%$ & $9.4 \%$ & $.0 \%$ \\
\hline & Otros & $7.7 \%$ & $3.3 \%$ & $2.9 \%$ & $.0 \%$ & $4.4 \%$ & $.0 \%$ \\
\hline & Ninguno & $30.8 \%$ & $25.4 \%$ & $28.6 \%$ & $25.9 \%$ & $36.5 \%$ & $50.0 \%$ \\
\hline
\end{tabular}

* El valor de chi-cuadrada es de 41.70 con 25 grados de libertad al nivel 0.018 .

Fuente: Elaboración propia con datos de la encuesta.

variables. Es decir, los posibles votantes encuestados no razonaron sobre el miedo como elemento importante al momento de considerar su decisión de votar o no votar, tampoco razonaron sobre la influencia de éste para orientar su sufragio.

Este hallazgo es importante ya que una de nuestras apuestas a través de este trabajo era la existencia de la relación entre el miedo generado por la crisis de inseguridad pública y el aumento o disminución del porcentaje de los votos obtenidos por los distintos partidos políticos. Los políticos, sus partidos, militantes o simpatizantes no apelaron al miedo generado por la criminalidad como estrategia para hacerse de votos. En este sentido se afirma que el miedo no influyó en el comportamiento del electorado.

\section{Conclusiones e implicaciones}

La violencia generada principalmente por las actividades de los grupos criminales originalmente ligados al narcotráfico se ha convertido en uno de los problemas más apremiantes que preocupan a los juarenses ya que las actividades criminales han aumentado significativamente en los últimos tres años. En este sentido, resulta atractivo analizar la relación existente entre el escenario social y el comportamiento del electorado. 
La primera y más importante de las conclusiones de este trabajo es que el contexto social enmarcado por la violencia configura la decisión en materia electoral. En nuestro caso, el fenómeno de la inseguridad pública orientó el comportamiento del electorado e influyó para que se presentaran los altos niveles de abstencionismo. La elección mayoritaria de no elegir gobernantes por la vía del voto es un rechazo a la falta de respuesta a una exigencia y necesidad elemental como es el derecho a vivir en un contexto seguro. En Ciudad Juárez el abstencionismo constituye un mensaje de frustración hacia gobiernos, candidatos y partidos políticos que se muestran lentos e ineficientes al momento de ofrecer alternativas de solución a problemas trascendentales.

Una segunda conclusión relevante es que los resultados esperados por el electorado y su relación con la evaluación de los gobernantes, candidatos y partidos políticos que el propio electorado elabora, genera dividendos a los mejor valorados. En este sentido, a pesar del alto abstencionismo presentado y de la insatisfacción generalizada hacia lo político, el Partido Revolucionario Institucional y sus candidatos fueron los actores que se posicionaron como los más capacitados para solucionar la crisis de inseguridad; además los gobiernos local y estatal emanados del PRI resultaron catalogados por los encuestados como los que habían desarrollado mejores resultados desde la administración pública. Esto, aunado a prácticas políticas tradicionales ${ }^{21}$ que no fueron objeto de estudio de este trabajo, coadyuvó a que el PRI retuviera la gubernatura del estado, la presidencia municipal, la sindicatura municipal y cinco de siete distritos electorales bajo disputa en el proceso electoral 2010.

La conclusión anterior da pie a una tercera. La crisis de inseguridad pública que azota a la ciudad desde enero de 2008 no genera votos para el partido opositor (PAN) por sí misma. En este sentido, bien se puede argumentar que la inseguridad pública puede convertirse en el agente persuasivo del comportamiento electoral más importante para la oposición, siempre y cuando este supuesto se respalde por buenas acciones de go-

\footnotetext{
${ }^{21}$ Se ha estudiado en varios trabajos algunas de las estrategias históricas que han producido resultados positivos al PRI en diversos procesos electorales, como la capacidad de movilización de las bases, la organización territorial, el voto corporativo, la operatividad electoral y, en algunos casos, el fraude.
} 
bierno y sobre todo por resultados generados por un ámbito de gobierno externo, en este caso el federal. El electorado del municipio de Juárez opinó sobre candidatos pertenecientes a Acción Nacional y al instituto político mismo como los menos capacitados para ofrecer alternativas de solución a la inseguridad pública y, aún más, mantiene la percepción de que el gobierno federal panista y su estrategia impulsada para reconstruir la ciudad no ha dado resultados.

De esta forma, las elecciones se convierten en una especie de plebiscito donde los electores evalúan el desempeño del partido gobernante en turno. Si esta evaluación es positiva o mejor de lo que representa la oposición, muchos de los votos favorecerán al partido en el gobierno. Si la evaluación es negativa, muchos de los votos se irán a la oposición.

Se puede afirmar que la inseguridad pública que se manifiesta en aumento en los niveles de criminalidad genera la emoción básica del miedo en el ser humano, en el caso bajo estudio, no orientó ni influyó en el comportamiento electoral. Los potenciales electores encuestados no razonaron de manera afirmativa sobre el miedo como factor importante al momento de considerar su decisión de votar o no votar, tampoco razonaron sobre la influencia de éste para orientar su sufragio hacia determinado partido político o hacia algún candidato en especial. Ésta constituye la cuarta conclusión de este trabajo.

Los anteriores hallazgos generan una implicación que resulta de utilidad para el estudio del impacto del contexto social en el comportamiento electoral. El instituto político, el gobierno y sus candidatos que sean más competentes para garantizar la superación de la crisis de inseguridad pública, o los que sean percibidos como los más capaces para enfrentar esta grave contingencia social, serán los que ganen las contiendas electorales, independientemente del papel que desarrollen como gobierno u oposición. Situación difícil de conseguir frente a un electorado escéptico, exigente y con tendencias históricas a favorecer el abstencionismo.

\section{Bibliografía}

Bauman Z. (2007), Miedo líquido. La sociedad contemporánea y sus temores, Paidós, Barcelona.

Bartels L. M. (1988), Presidential Primaries and the Dynamics of Public Choice, Princeton University Press. Princeton, Nueva Jersey. 
Blais A. (2006), "What Affects Voter Turnout?”, Annual Review of Political Science, 6: 111-125.

Butler, D. y D. Kavanagh (1997), The British General Election of 1997, Macmillan, Londres.

Campbell, J. E., L. L. Cherry y K. A. Wink (1992), “The Convention Bump”, American Politics Quarterly, 20: 287-307.

Chomsky, N. (2006), Failed States. The Abuse of Power and the Assault of Democracy, Holt Paperbacks, Nueva York.

Cooper, E. M. (1947), "The Evasion of Propaganda: How Prejudiced People Respond to Anti Prejudiced Propaganda”, The Journal of Psychology, 23: 1525.

Downs, A. (1957), Teoría económica de la democracia, Aguilar, Madrid.

Enelow, J. y M. J. Hinich (1984), The Spatial Theory of Voting: An Introduction, Cambridge University Press, Cambridge.

Fan, D. (1988), Predictions of Public Opinion from the Mass Media, Greenwood, Nueva York.

Fernández, L. y A. Aguilera ( 2002), Violencia y elecciones en Santander, recuperado el 03 de mayo del 2010 de <http://editorial.unab.edu.co>.

Festinger, L. (1957), A Theory of Cognitive Dissonance, Stanford University Press, Stanford, California.

Foucault, M. (1976), Vigilar y castigar, Siglo XXI, Buenos Aires.

Foreign Policy (2010), “12 Degrees of Failure. ¿How does a Weak State become a Failed State?”, Foreign Policy, julio-agosto.

Geer J. G. (1988), "The Effects of Presidential Debates on The Electorate's Preferences for Candidates", American Politics Quarterly, 16: 486-501.

Graber D. A. (1980), Mass Media and American Politics, Congressional Quarterly Press, Washington, DC.

Heath, A., R. Jowell, J. Curtice y S. Witherspoon (1991), Understanding Political Change: The British Voter 1964-1987, Pergamon, Oxford.

Hobbes T. (1987), Del ciudadano y Leviatán, estudio preliminar y antología de Enrique Tierno Galván, Traducción de Enrique Tierno Galván y M. Sánchez Sarto, Tecnos, Madrid.

Instituto Estatal Electoral de Chihuahua (2010), Resultados del proceso electoral Chihuahua 2010.

Instituto Mexicano de la Juventud (2005), Encuesta Nacional de la Juventud 2005.

Instituto Nacional de Estadística, Geografía e Informática (2010), Resultados preliminares del Censo de Población y Vivienda. (2009), Encuesta Nacional de Ocupación y Empleo.

Iyengar, S., M. Peter y D. Kinder (1982), "Experimental demonstration of the not-so-minimal, consequences of television news programs", American Political Science Review, 76: 848-858. 
Kappler, J. (1960), The Effects of Mass Communication, Glencoe, IL, Free Press.

Kreps, D. M. (1990), A Course in Microeconomic Theory, Princeton, Princeton University Press.

Lazarfeld, P. F., B. Berelson y H. Gaudet (1944), The people's Choice: How the Voter Makes Up His Mind in a Presidential Campaign, Columbia University Press, Nueva York.

Lehoucq, F. (2009), "Ingreso y cálculos electorales en 2006", Política y gobierno, CIDE, México, volumen temático 2009: 107-136.

Lohr, S. (2000), Muestreo: diseño y análisis, International Thomson Editores, México.

Maquiavelo, N. (2009), El príncipe, Ediciones Gandhi, México.

McCombs M. y L.D. Shaw (1972), "The Agenda-Setting Function of the Mass Media”, Public Opinion Quaterly, 36: 176-187.

Mendelson, P. F. y G. J. O’Keefe (1976), The People Choose a President, Praeger, Nueva York.

Mendoza, G. (2006), "La mediatización y la construcción de opinión pública", Gaceta Universitaria, 418, 12 de diciembre.

Noélle-Neuman, E. (1983), "The Effects of Media on Media Effects Research", Journal of Communication, 33: 157-165.

Norris, P., J. Curtice, D. Sanders M. Scammell y H.A. Semetko (1999), On Message. Communicating the Campaign, Sage, Beverly Hills.

Organizacion de las Naciones Unidas (2009), Encuesta Internacional sobre Criminalidad y Victimización.

Padilla, H. (2010), Para mí es lo mismo. Causas del abstencionismo en Ciudad Juárez, Universidad Autónoma de Ciudad Juárez, Instituto Estatal Electoral Chihuahua, Chihuahua.

Page, Shapiro y Dempsey (1987) “What Moves Public Opinion?”, American Political Science Review, 81: 23-44.

Paniagua, A. (2009), Para los que no creyeron ... y para los que siguen sin creer... El discurso como herramienta de poder entre la relación Estado-sociedad-crimen organizado en Ciudad Juárez. Un análisis, conferencia parte de la VII Bienal Iberoamericana de la Comunicación, Chihuahua.

Patterson, T. (1980), The Mass Media Election, Praeger, Nueva York.

Robin, C. (2004), Fear: The History of a Political Idea, Oxford University Press, Nueva York.

Sartre, J. (1978), La trascendencia del ego, Síntesis, Colombia.

Sanders, D. (1997), "Voting and the Electorate", en P. Dunleavy, A. Gamble, I. Holiday y G. Peele (eds.), Developments in British Politics 5, Macmillan, Londres.

Sandoval L. E. (2008), Determinantes del comportamiento electoral de los municipios colombianos en zonas de conflicto interno durante 1997-2006, recuperado el 24 de marzo de la pagina <http://www.rlcu.org.ar $>$. 
Scheaffer, R., W. Mendenhall y L. Ott (1987), Elementos de muestreo, Iberoamericana, México.

Seguridad, Justicia y Paz. (2010), Ciudad Juárez por segundo año consecutivo la ciudad más violenta de mundo, boletín de prensa, lunes 11 de enero.

Serrano, M. y M. Toro (2005), "Del narcotráfico al crimen transnacional organizado en América Latina”, en Mats Berdal y Mónica Serrano (coord.) Crimen transnacional organizado y seguridad internacional. Cambio y continuidad, Fondo de Cultura Económica, México.

Universidad Autonoma de Ciudad Juárez (2010), Observatorio de seguridad y convivencia ciudadanas del municipio de Juárez, documento de trabajo. 